\title{
Manganese-Enhanced Magnetic Resonance Imaging for Detection of Vasoactive Intestinal Peptide Receptor 2 Agonist Therapy in a Model of Parkinson's Disease
}

\author{
Katherine E. Olson ${ }^{1}$ - Aditya N. Bade ${ }^{1}$. Charles R. Schutt ${ }^{1}$. Jingdong Dong ${ }^{2}$. \\ Scott J. Shandler ${ }^{3} \cdot$ Michael D. Boska $^{1,2} \cdot$ R. Lee Mosley ${ }^{1} \cdot$ Howard E. Gendelman $^{1}$ • \\ Yutong Liu ${ }^{1,2}$
}

Published online: 21 June 2016

(C) The American Society for Experimental NeuroTherapeutics, Inc. 2016 developed as a biomarker tool to monitor neurotherapeutic responses that are relevant to common neurodegenerative disorders used to improve disease outcomes.

Key Words Magnetic resonance imaging (MRI) · manganese enhanced MRI (MEMRI) · MPTP · inflammation · glial activation $\cdot$ neuroprotection

\section{Introduction}

Progressive nigrostriatal degeneration and its associated neuroinflammation contribute to the pathogenesis of Parkinson's disease (PD). Although the cause of PD remains unknown, aberrant innate and adaptive immune responses direct the tempo of nigral neuronal loss [1-3]. Associated immune activation is propagated through the accumulation and extracellular release of $\alpha$-synuclein $(\alpha-$ syn), an endogenous self-protein [4]. Such extracellular release of aggregated and post-translationally modified $\alpha$-syn results in glial activation, which, in turn, affects the breakdown of immune tolerance. These pathological events underlie progressive dopaminergic neuronal loss and microglial activation [5-7]. Indeed, neuronal loss is accompanied by robust microgliosis, astrocytosis, and release of proinflammatory neurotoxic mediators [8-11]. Recent works from our own laboratories have served to highlight the role of effector $\mathrm{T}$ cells (Teffs) and regulatory $\mathrm{T}$ cells (Tregs) in attenuating such neuroinflammatory response cascades [12,13]. The balance between effector $\mathrm{T}$ cells and Treg activities leads to respective degenerative or protective effects in the brain, depending on defined T-cell polarity and cell phenotype [2,12,14-17]. Immune profiles performed in
Department of Pharmacology and Experimental Neuroscience, University of Nebraska Medical Center, Omaha, NE, USA

2 Department of Radiology, University of Nebraska Medical Center, Omaha, NE, USA

3 Longevity Biotech, Philadelphia, PA, USA 
patients with PD support a functional balance between the cells as circulating immunocyte profiles demonstrate increased proinflammatory profiles and decreased Treg function [18]. Such aberrations serve to change the brain's microenvironment, where a proinflammatory dominance ensues. These notions are further supported by preclinical studies performed in a 1-methyl-4-phenyl-1,2,3,6-tetrahydropyridine (MPTP) model of PD where intervention by immune modulation serves to protect dopaminergic neurons within the nigrostriatal pathway $[13,19]$.

Mobilizing specific regulatory T-cell adaptive immune responses affects microglial reactivity and protect against nigrostriatal degeneration $[12,13,19]$. The use of immunomodulatory compounds, such as granulocyte macrophage colony-stimulating factor (GM-CSF) and vasoactive intestinal peptide (VIP), shifts immune phenotypes and functions from neurotoxic to neuroprotective regulatory responses [13,19-22]. Specifically targeting VIP receptor 2 (VIPR2) with a modified VIP analog (referred to as LBT-3627) has shown robust neuroprotective activities in MPTP-intoxicated mice [19]. LBT-3627 is a receptor-selective and metabolically stable agonist, as well as - like GM-CSF - a potent inducer of Treg. LBT-3627 attenuates microglial activation and induces endogenous GM-CSF leading to increased Treg functional activities and potentiation of dopaminergic neuronal sparing [19]. LBT-3627 has potent neuroprotective and immunemodulating effects, eliciting a dose-dependent sparing of dopaminergic neurons, a substantial decrease in microglial response at a time of peak inflammation, decreases in proinflammatory cytokine production, and significant changes in genes associated with T-cell and microglial populations. In these studies, we also assessed the abilities of a scrambled peptide, a VIPR1-specific agonist, and a VIPR antagonist to elicit a protective response under the same conditions. Upon extensive evaluation, we found greater neuroprotective and immune modulating abilities using LBT-3627. Moreover, its use may not be associated with untoward adverse events [23]. Thus, we chose to further assess the neurotherapeutic capacities of LBT-3627 in a preclinically relevant model using bioimaging techniques. We employed manganese (Mn)-enhanced magnetic resonance imaging (MEMRI) as a noninvasive imaging biomarker to follow disease progression and to assess the abilities of LBT-3627 therapies to slow neurodegenerative activities. However, even with promising results, a potential downside of its use rests in the fact that $\mathrm{Mn}$ exposure can be linked parkinsonism in humans $[24,25]$. Thus, owing to the toxicity of $\mathrm{Mn}$, MEMRI has only been utilized in animal studies. The feasibility of using MEMRI in humans is still under investigation. Nonetheless, we now show that MEMRI is an effective noninvasive tool that can be developed as a means to follow immune-modulatory neurotherapies in experimental models for preclinical testing.

\section{Methods}

\section{Animals, Drug treatment, and MPTP Intoxications}

Male C57BL/6J mice, 6-8 weeks old (The Jackson Laboratory, Bar Harbor, ME, USA) were used in all studies. Mice were pretreated with LBT-3627 (Longevity Biotech, Inc., Philadelphia, PA, USA), a VIPR2 agonist, at intraperitoneal dosages of $15 \mu \mathrm{g}$ or $45 \mu \mathrm{g}$ daily for 5 days prior to MPTP intoxication. On the sixth day, mice received 4 subcutaneous injections of vehicle [Dulbecco's phosphate-buffered saline (DPBS), $10 \mathrm{ml} / \mathrm{kg}$ body weight] or MPTP-HCl (SigmaAldrich, St. Louis, MO, USA) at $16 \mathrm{mg}$ MPTP (free base)/ $\mathrm{kg}$ body weight in DPBS, with each injection given every $2 \mathrm{~h}$. MPTP safety precautions were followed according to published safety and handling protocol [26,27]. All animal procedures used in this report were approved by the Institutional Animal Care and Use Committee of the University of Nebraska Medical Center and were in agreement with the guidelines set by the National Institutes of Health.

\section{MEMRI}

A $50 \mathrm{mM}$ solution of $\mathrm{MnCl}_{2} \cdot 4 \mathrm{H}_{2} \mathrm{O}$ (Sigma-Aldrich) in $0.9 \% \mathrm{w} / \mathrm{v} \mathrm{NaCl}$ (Hospira, Lake Forest, IL, USA) was used for MEMRI. For MEMRI examinations, $60 \mathrm{mg}(0.3 \mathrm{mmol}) / \mathrm{kg}$ $\mathrm{MnCl}_{2}$ solution was administered intraperitoneally at 48 and $24 \mathrm{~h}$ prior to initiation of examination. The fractioned $\mathrm{MnCl}_{2}$ administration scheme (1 i.p. dose per day for 2 days) were used to control uneven $\mathrm{Mn}^{2+}$ distribution caused by a variety reasons, including differences in extracellular and intracellular $\mathrm{Mn}^{2+}$ concentrations, anterograde transport through neurons, and injection variability. This design allows $\mathrm{Mn}^{2+}$ to reach a stable distribution as previously published by others and our laboratory $[28,29]$. The period of stable distribution allows for reproducible and even distribution of signal during MRI [28-34]. For studies performed 2 days post-MPTP treatment, the first $\mathrm{MnCl}_{2}$ was injected $4 \mathrm{~h}$ after MPTP/DPBS administration. After lesion formation (day 5), MEMRI examinations were performed on a staggered basis for days 7-16 postMPTP/DPBS treatment and were binned together (hereafter designated as day-7 examinations). As only 4 MEMRI examinations could be completed in 1 day, staggering examinations using 1 subject from each of 4 treatment groups ( 4 total/day) were necessary to minimize between group variability.

For both day-2 and day-7 examinations, mice were scanned in a $7 \mathrm{~T} / 21 \mathrm{~cm}$ MRI scanner (Bruker, Billerica, MA, USA). Animals were anesthetized by inhalation of isoflurane in $100 \%$ oxygen and maintained at $40-80$ breaths/minute. Anesthetized mice were scanned using a $T_{1}$ mapping sequence (fast spin echo with variable repetition time from $0.4 \mathrm{~s}$ to $10 \mathrm{~s}, 12$ slices, slice thickness $0.5 \mathrm{~mm}$, in-plane resolution $0.1 \times 0.1 \mathrm{~mm}^{2}$ ) and $\mathrm{T}_{1}$-weighted $\left(\mathrm{T}_{1}\right.$-wt) MRI (FLASH, 
repetition time $20 \mathrm{~ms}$, flip angle $20^{\circ}$, 3-dimensional isotropic resolution $0.1 \times 0.1 \times 0.1 \mathrm{~mm}^{3}$ ). After data acquisition, the mice were euthanized and tissues removed for immunohistological studies. $T_{1}$ and $T_{1}$-wt scans were performed prior to MPTP treatment, and the acquired image served as baseline data for the calculation of Mn-mediated signal enhancement.

\section{MRI Data Processing}

Brain volumes in the $T_{1}$-wt images were first extracted using an in-house MATLAB program based on the level sets method. The brain images were then registered to the MRI-based mouse brain atlas (Laboratory of Neuro Imaging, University of California Los Angeles) using affine transformation first, and then nonlinear transformation (DiffeoMap; John Hopkins University, Baltimore, MD, USA). The baseline and post$\mathrm{MnCl}_{2}$ injection, $\mathrm{T}_{1}$-wt images were calibrated using $\mathrm{T}_{1}$ values to minimize the MRI system variations between the baselines and post- $\mathrm{MnCl}_{2}$ injection scans as described [35]. Signal enhancement maps were calculated as follows: $\left(S_{M n}-\mathrm{S}_{\mathrm{bl}}\right) / \mathrm{S}_{\mathrm{bl}}$, where $S_{M n}$ and $S_{b l}$ are $\mathrm{T}_{1}$-wt signal intensity of post- and pre- $\mathrm{MnCl}_{2}$ injection, respectively. The MEMRI enhancement maps were segmented automatically into 16 brain regions/subregions using the atlas. The enhancements in the regions were measured, and 1 -way analysis of variance (ANOVA) performed to examine the significance of enhancement change among groups. A pixel-by-pixel comparison was also performed among groups using 1-way ANOVA. A pixel on a brain image was compared with corresponding pixels on the same locus on other brain images.

\section{Tissue Acquisitions and Immunohistochemistry}

Following MEMRI scanning, mice were terminally anesthetized and transcardially perfused with DPBS followed by $4 \%$ paraformaldehyde/DPBS (Sigma-Aldrich) at day 2 or days $7-$ 16 post-MPTP intoxication (referred to as day 7). Whole brains were harvested, processed, and flash-frozen to assess dopaminergic neuronal survival and glial reactivity in the substantia nigra (SN) as previously described [36]. Frozen midbrain sections $(30 \mu \mathrm{m})$ were immunostained for either tyrosine hydroxylase (TH) (anti-TH, 1:2000; EMD Millipore, Billerica, MA, USA), Mac-1 (anti-CD11b, 1:1000; AbD Serotech, Raleigh, NC, USA), or glial fibrillary acidic protein (anti-GFAP, 1:1000; Dako, Carpinteria, CA, USA). Within the SN, numbers of $\mathrm{GFAP}^{+}$cells $/ \mathrm{mm}^{2}$, Mac$1^{+}$cells $/ \mathrm{mm}^{2}$, total numbers of $\mathrm{TH}^{+} \mathrm{Nissl}^{+}$(dopaminergic neurons), and total numbers of $\mathrm{TH}^{-} \mathrm{Nissl}^{+}$(nondopaminergic neurons) were estimated using stereological analysis via Stereo Investigator software with the optical fractionator module
(MBF Bioscience, Williston, VT, USA), as previously described [13].

\section{Statistical Analysis}

All immunohistochemical values are expressed as mean \pm SEM. Differences in between-group means were analyzed using ANOVA followed by Fisher's least significant difference post hoc test (GraphPad Software, Inc., La Jolla, CA, USA).

\section{Results}

\section{MEMRI Signal Enhancement}

Peak neuroinflammation and rate of neuronal death as measured by reactive microglia occurs 2 days post-MPTP intoxication [9,37]. We chose to examine the ability of MEMRI to detect changes between MPTP and DPBS controls during this time. MEMRI provides information relevant to assessments of neural anatomy, function, and connectivity [38,39]. $\mathrm{Mn}^{2+}$ enters neurons through voltage-gated calcium $\mathrm{Ca}^{2+}$ channels, owing to its similarities with $\mathrm{Ca}^{2+}$, linking $\mathrm{Mn}^{2+}$ accumulation to MEMRI signal intensity [40]. Additionally, in prior studies, we demonstrated that MEMRI signals are increased during neuroinflammation due to elevated neuronal $\mathrm{Mn}^{2+}$ uptake and accumulation through astrocyte activation $[35,41]$. Thus, comparisons of pretreatment scans and day-2 or day-7 DPBS post-treatment scans demonstrated that $\mathrm{MnCl}_{2}$ administration yielded enhanced signal intensities (hereafter designated as MEMRI signal) (Figs. 1 and 2). Previous reports of Mnenhanced $\mathrm{T}_{1}$-wt signaling confirm these results $[35,41]$. To minimize between-group by day variability, one subject from each of 4 treatment groups were assessed every day on days 7-16; a time after MPTP-induced lesion forms [37]. Linear regression of all dependent experimental parameters for an effect of day showed no significant effects on any dependent variable except for MEMRI signal intensities and changes in intensities for mice treated with MPTP and $15 \mu \mathrm{g}$ LBT-3627 $\left(r^{2}=0.64, p=0.0176\right)$. However comparison of signal levels obtained on days 7-10 with those obtained on days 11-14 showed no significant differences by $t$ test $(p=0.1091)$. Thus, data acquired on days $7-16$ were binned as 1 time point and are hereafter designated as day-7 data.

Using baseline $\mathrm{T}_{1}$-wt images, we prepared mean MEMRI signal enhancement maps for day 2 and day 7 acquisitions (Figs. 1a and 2a). Following bioimaging, results indicate that on day 2 following MPTP intoxication (Fig. 1a), the MEMRI signal in the SN was diminished (column 3, row 4), while that in the striatum was increased (column 3 , rows 1 and 2). Pixels with significant signal changes $(p<0.05)$ were superimposed on anatomical structures to form a $p$-value map (Fig. 1b). 


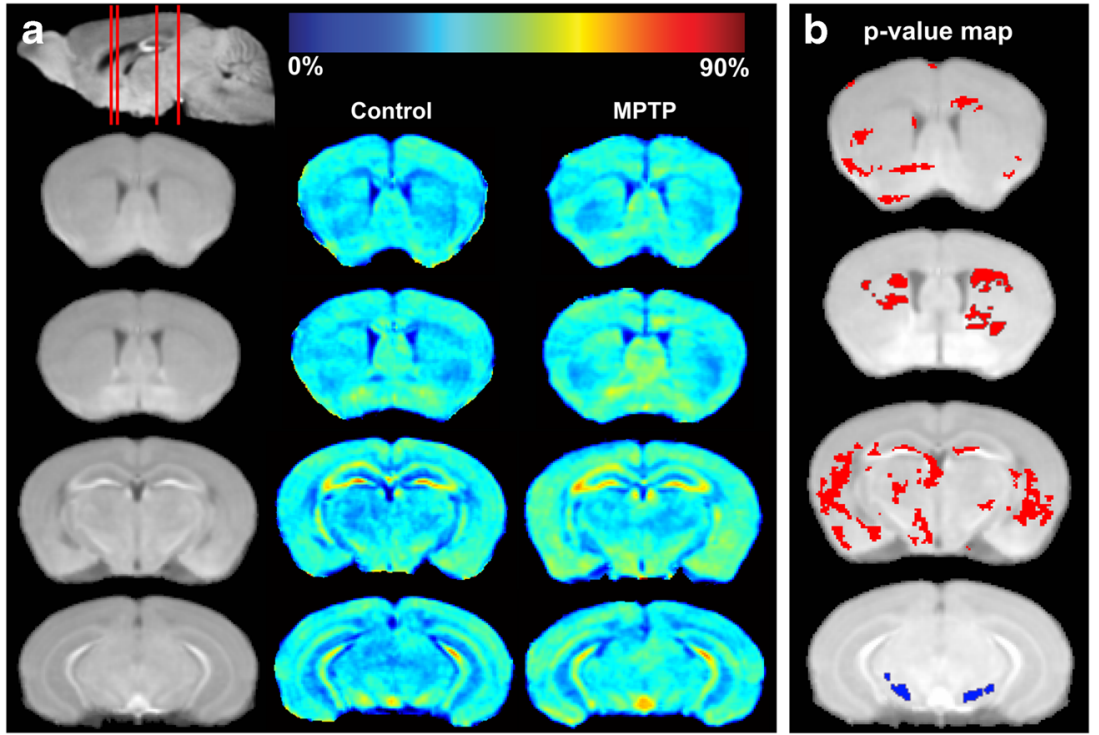

Fig. 1 Comparison of MEMRI signal enhancement between controls and MPTP-intoxicated mice at day 2 post-MPTP. (A) MEMRI enhancement maps. The first column (from left) shows coronal slices of the averaged MEMRI of control mice $(n=5)$ as an anatomical reference. The sagittal slice (upper left) shows respective coronal slices (red lines from rostral to caudal) that correspond to images in rows 1-4 showing sections with striatum (rows 1 and 2) or midbrain (rows 3 and 4). The second column shows the average enhancement in control mice on the coronal slices. The third column represents the average enhancement of
MPTP-intoxicated mice $(n=6)$. The color bar for the enhancement maps is at the top of the figure. Dark blue color ( $0 \%)$ means no change in enhancement from $\mathrm{Mn}^{2+}$ compared with preinjection signal intensity. Dark red color represents $90 \%$ signal increase compared with preinjection signal intensity. (B) Statistical comparison of MEMRI enhancement between control and MPTP-intoxicated mice. Pixels with a significant increase $(p<0.05)$ in signal intensity are indicated in red, with decreases indicated in blue. Pixel changes are overlaid onto the averaged brain image for visualization
Significantly decreased MEMRI signals were found primarily within the SN (row 4). Striatum contained the most pixels of significantly increased signals (rows 1 and 2). A region-byregion comparison facilitated by the MRI-based atlas indicated the same findings (data not shown). A number of pixels on cerebral cortex and hippocampus also showed significant enhancement increases. However, the comparison did not find enhancement differences between the 2 groups. Comparison of subregions of hippocampus and cortex was not performed because the MRI-based mouse brain atlas employed for enhancement map segmentation does not identify these subregions.

Next, we assessed MEMRI signal after lesion formation in mice pretreated with LBT-3627. By day 7, the major inflammatory condition associated with reactive microglia in MPTP is essentially resolved, leaving only astrogliosis and the nigrostriatal lesion to be observed [9]. In contrast to day 2, by day 7 MEMRI signals for DPBS controls and MPTPintoxicated mice were similar in most brain regions, including SN (column 3, rows 3 and 4). Signal intensity within the SN was significantly decreased in low-dose LBT-3627-treated mice compared with MPTP-intoxicated mice (columns 4 and 5, rows 3 and 4). The pixel-wise $p$-value map also showed the most pixels on $15 \mu \mathrm{g}$-treated with lower signal compared with MPTP-treated mice (Fig. 2b). Mice treated with the higher dose of LBT-3627 also had lower MEMRI signal when compared with MPTP; however, it did not reach significance. The lack of significance is potentially due to the high variation of signal intensity and low number of animals. In accordance with our previous dose-response findings, a $45-\mu \mathrm{g}$ dose appears to elicit an extremely variable response, indicating that we may be approaching a therapeutic threshold [19]. In addition, both cerebral cortex and hippocampus included pixels that had significantly lower signal (Fig. 2b). However, the brain atlas-based regional comparison did not find a significant difference between 15- $\mu$ g-treated and MPTP mice in these 2 regions.

\section{Immunohistology}

In order to assess the ability of MEMRI to detect neuronal loss and/or inflammation during MPTP, we carried out immunohistological evaluations to determine dopaminergic neuron numbers, microglial reactivity, and astrogliosis. As expected, $\mathrm{TH}^{+} \mathrm{Niss}^{+}$dopaminergic neurons were lost following MPTP intoxication (Fig. 3), whereas $\mathrm{THNiss}^{+}$neurons remained unchanged. At day 2, neuron numbers significantly dropped from $8020 \pm 379$ to $3938 \pm 317$, equivalent to a $51 \%$ loss (Fig. 3a). At day 7, MPTP intoxication markedly reduced neuron levels to $42 \%$, with an increased loss compared with day 2 (Fig. 3b). Both the low and high dose of LBT-3627 significantly spared dopaminergic neurons, resulting in $62 \%$ and $64 \%$ neuron 

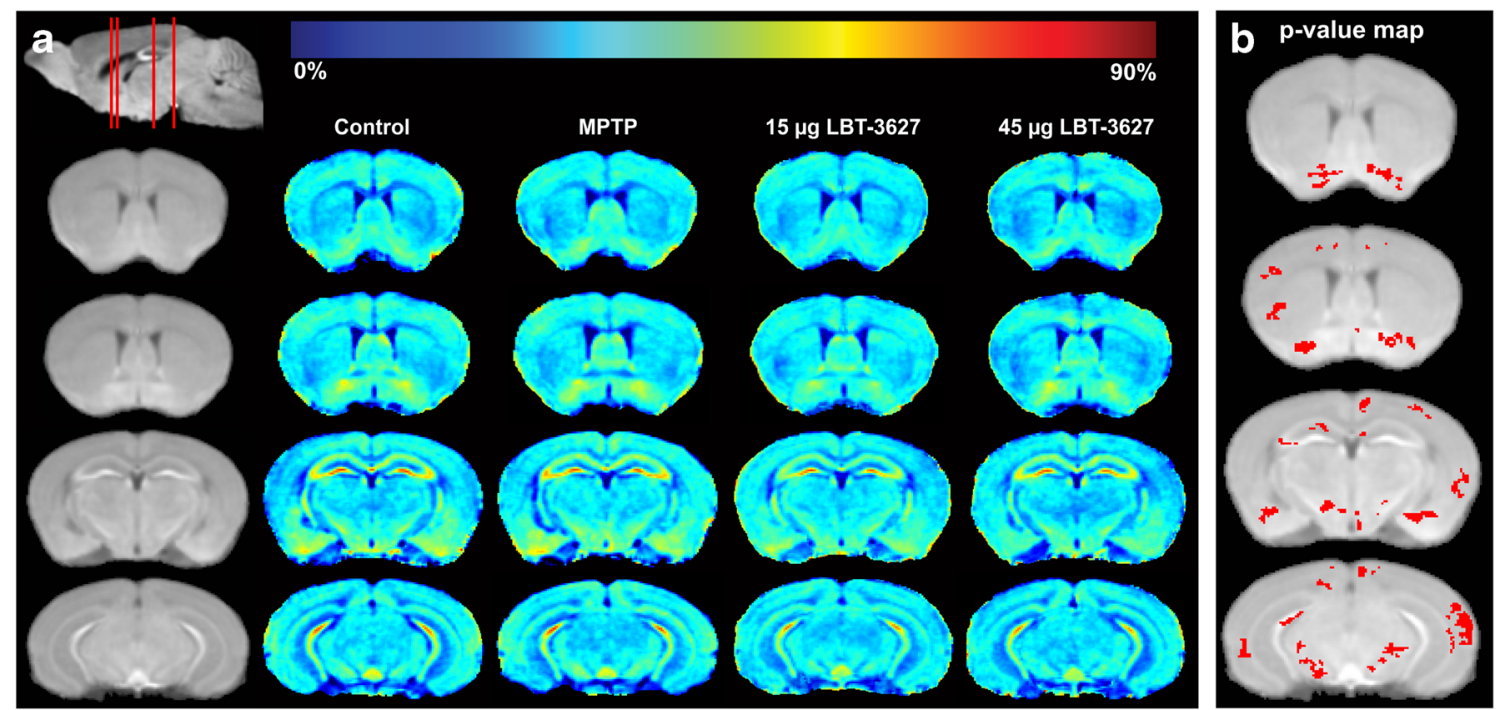

Fig. 2 Comparison of MEMRI enhancement between controls and MPTP-intoxicated mice at day 7. (A) MEMRI enhancement maps. The first column (from left) shows coronal slices of the averaged MEMRI of control mice $(\mathrm{n}=5)$ as an anatomical reference. The sagittal slice (upper left) shows respective coronal positions (red lines). The second column shows the average enhancement in control mice on the coronal slices. The third column represents the average enhancement of MPTP-intoxicated mice $(n=6)$. The fourth column represents the average enhancement of MPTP-intoxicated mice treated with $15 \mu \mathrm{g}$ LBT-3627. The fourth column represents the average enhancement of MPTP-intoxicated mice treated with $45 \mu \mathrm{g}$ LBT-3627. The color bar for the enhancement maps is at the top of the figure. Dark blue color (0\%) means no change in enhancement from $\mathrm{Mn}^{2+}$ compared with preinjection signal intensity. Dark red color represents $90 \%$ signal increase compared to preinjection signal intensity. (B) Statistical comparison of MEMRI enhancement between MPTPintoxicated mice and MPTP-intoxicated mice treated with $15 \mu \mathrm{g}$ LBT3627 . It shows the pixels (red color) with significant decrease $(p<0.05)$ in signal intensity overlaid onto the averaged brain image survival compared with DPBS alone. At 2 and 7 days postMPTP, reactive microglial populations were also assessed (Fig. 4). When compared with day 2 DPBS controls, MPTP significantly increased reactive microglial cell numbers from $2.8 \pm 0.3 \mathrm{cells} / \mathrm{mm}^{2}$ to $46 \pm 5$ cells $/ \mathrm{mm}^{2}$, indicating an inflammatory response associated with MPTP intoxication (Fig. 4a). By day 7, there was a significant decrease in observed microglial reactivity, when compared with MPTP-treated mice (Fig. 4b). The number of reactive microglia decreased by $48 \%$ from day 2 to day 7, indicating a resolution of inflammation. LBT-3627 treatment also led to a small decrease in reactive microglial numbers, standing at $15.18 \pm 3$ cells $/ \mathrm{mm}^{2}$ and $14.96 \pm 2.7$ cells $/ \mathrm{mm}^{2}$ with both a low and high dose, respectively. However, the magnitude of changes in microglial activation at day 7 is small, which is expected in this model. With the acute MPTP model of PD, the reactive microglial response generally subsides by day 7 ; thus, the minute differences observed between groups is in accordance with what has been previously demonstrated in the literature $[8,9]$. In addition, GFAP-immunoreactive cells were assessed following MPTP intoxication at both time points, as well as following low- and high-dose LBT-3627 pretreatment (Fig. 5). At day 2, MPTP intoxication led to a nonremarkable increase in astroglial response (Fig. 5a). However, by day 7, the increase was highly significant. Compared with DPBS, MPTP intoxication noticeably increased GFAP reactivity by nearly 6-fold from $12 \pm 2$ cells $/ \mathrm{mm}^{2}$ to $61 \pm 8$ cells $/ \mathrm{mm}^{2}$. Both doses of LBT-3627 pretreatment attenuated astroglial response by significantly decreasing GFAP reactivity by $\sim 50 \%$ when compared with MPTP treatment alone (Fig. 5b).

In developing MEMRI as a potential therapeutic biomarker, we sought to correlate signal intensity change with the immunohistological findings (Fig. 6). When comparing dopaminergic neuron $\left(\mathrm{Th}^{+} \mathrm{Niss}^{+}\right)$number and signal intensity, data was found to be associated in all treatment arms except for MPTP-treated mice at day 7 that did not receive LBT-3627 treatment (Fig. 6a). Microglial reactivity seemed to be inversely related to signal enhancement, indicating that microglial response over time does not contribute to the MEMRI signal strength (Fig. 6b). As expected, correlation analysis of combined day 2 and day 7 data points revealed a positive relationship between signal intensity and $\mathrm{TH}^{+}$neuronal number $(r=0.36, p=0.0208)$, and a negative relationship between signal intensity and $\mathrm{Mac}-1^{+}$microglia cell numbers $(r=-0.42, p=0.0066)$, indicating that increases in signal intensity correlate with increased neuron number and decreased microglial response. Interestingly, for $\mathrm{GFAP}^{+}$staining, there was not a significant correlation observed when including all data points. Conversely, when comparing data on day 2 alone, changes in $\mathrm{GFAP}^{+}$staining was negatively related to changes in signal intensity in MPTP-treated groups $(r=-0.74$, $p=0.0092$ ), as shown in Fig. 6c. At day 2, when MEMRI signal intensity was significantly decreased from DPBS control levels, GFAP ${ }^{+}$astrocyte numbers were also low, with no 
a
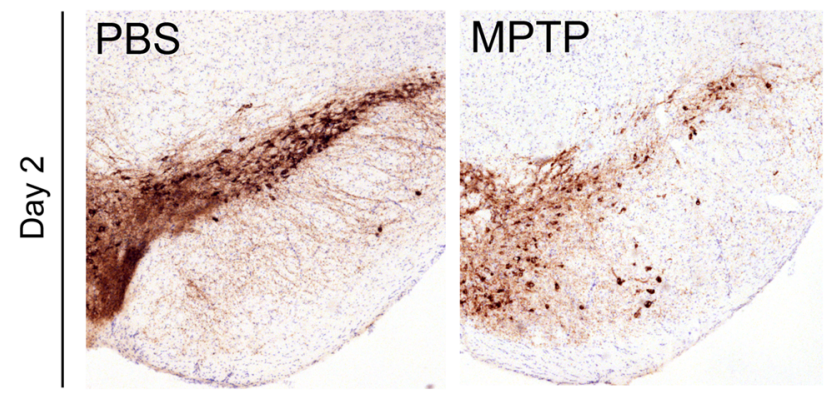

b

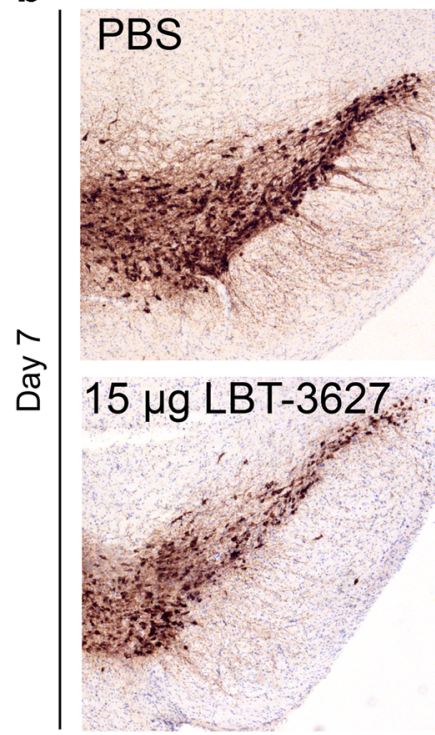

MPTP
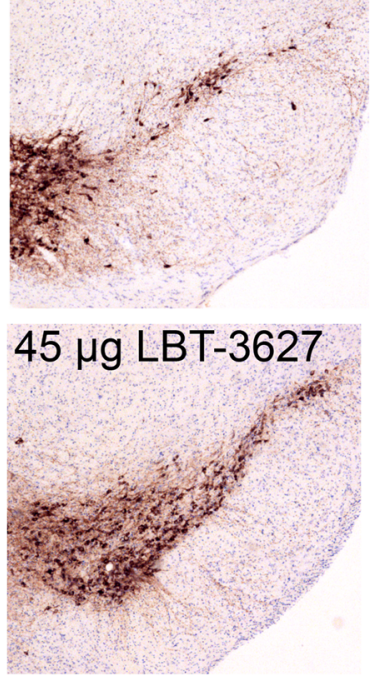

Day 2

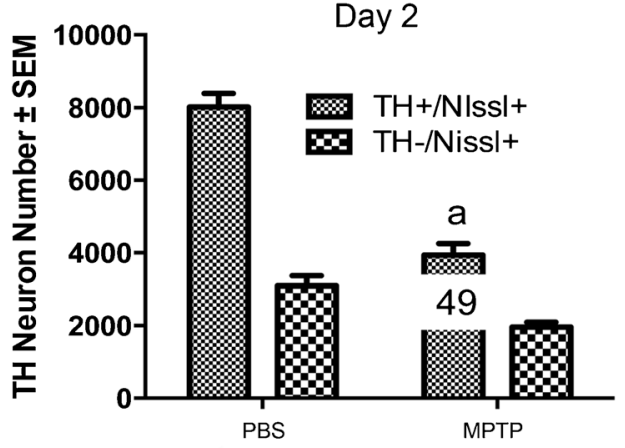

Day 7

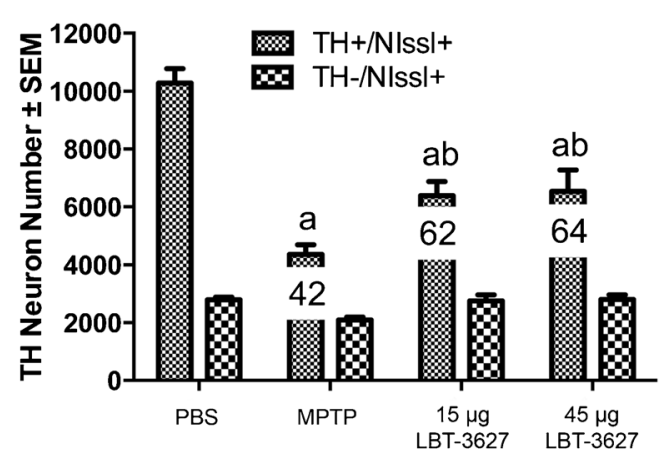

Fig. 3 Assessment of neuronal survival following MPTP intoxication and LBT-3627 pretreatment. (A) Photomicrographs of $\mathrm{TH}^{+} \mathrm{Niss}^{+}$ neurons in the substantia nigra $(4 \times)$ in mice treated with PBS or MPTP, followed by quantification of $\mathrm{TH}^{+} \mathrm{Nissl}^{+}$and $\mathrm{TH} \mathrm{Nissl}^{+}$neuronal counts. (B) Photomicrographs of $\mathrm{TH}^{+} \mathrm{Niss}^{+}$neurons within the $\mathrm{SN}$ following treatment with MPTP or LBT-3627 pretreatment of doses of $15 \mu \mathrm{g}$ or $45 \mu \mathrm{g}$. Quantification of $\mathrm{TH}^{+} \mathrm{Niss}^{+}$and $\mathrm{TH}^{\mathrm{Niss}}{ }^{+}$neurons is shown in

significant change observed between DPBS and MPTP treatment. However, by day 7, MEMRI signal intensity corresponded with significantly higher $\mathrm{GFAP}^{+}$reactivity, indicating that the increased signal may be due to an activation of astrocytes, which outweighed the expected decreased signal due to the significant neuronal loss seen 7 days after the formation of an MPTP-mediated lesion. Likewise, in the mice pretreated with LBT-3627, the decrease in MEMRI signal was likely due to the loss of neurons from MPTP. Also, there was an insufficient astroglial response to restore the MEMRI signal to that of the DPBS controls.

\section{Discussion}

Progressive neuronal degeneration underlies the pathobiology of a broad range of neurodegenerative diseases that the right panel. All sections were immunostained with antityrosine hydroxylase, color was generated with diaminobenzidine, and sections were counterstained with thionin. Differences in means ( \pm SEM, $n=5-8$ ) were determined by analysis of variance followed by Fisher's least significant difference post hoc test where $p<0.05$ compared with (a) PBS or (b) MPTP

include PD [42]. While the causes of most neurodegenerative diseases are not known, the relationship between neurodegeneration and immune system activation is clear. Therapies are consistently being developed and tested to modulate or transform the immune system with the intention to clear misfolded, aggregated, and nitrosylated proteins, as well as to restore the damaged central nervous system microenvironment [43]. Amongst these therapies, there are agents that can change a dominant effector phenotype to one that promotes immune tolerance and is regulatory, anti-inflammatory, and neuroprotective. One such candidate is LBT-3627. This drug has shown robust neuroprotective potential in MPTP-intoxicated animals to attenuate neuroinflammatory responses while affecting Treg function [19]. Despite such effects, broad obstacles still lie ahead in bringing the medicines to human use. With this in mind, our laboratory has pioneered preclinical 
a

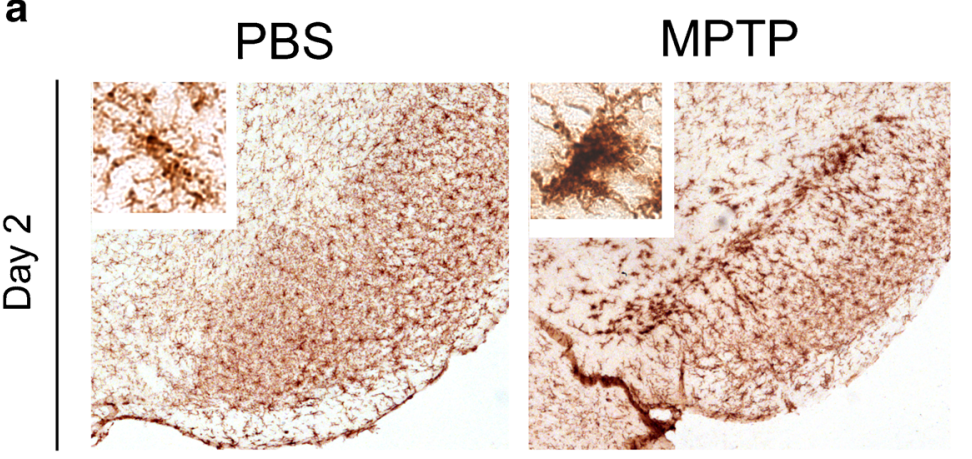

b

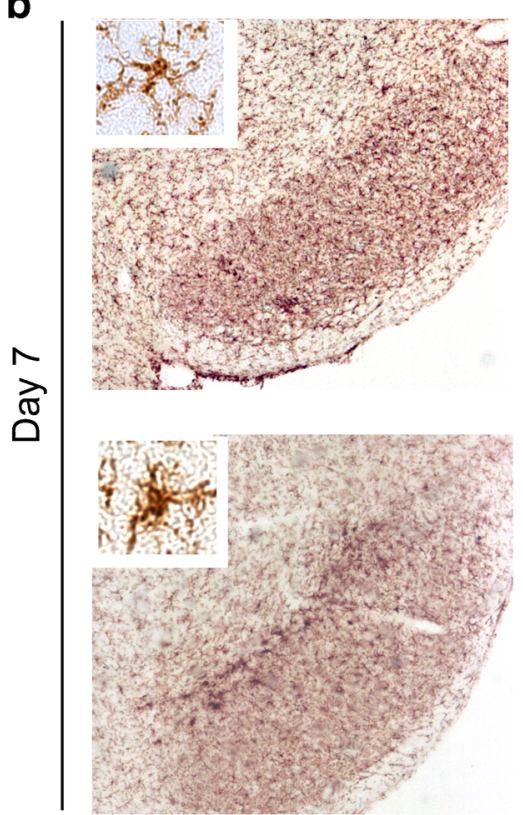

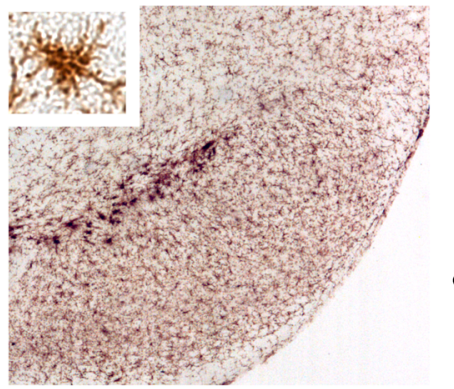

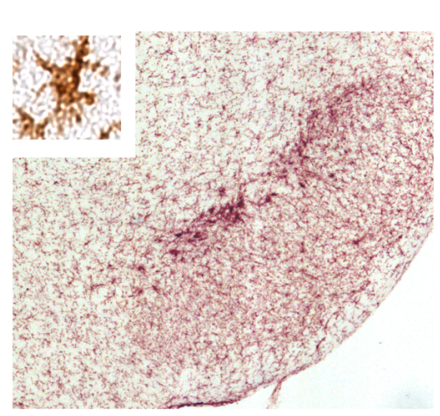

Day 2

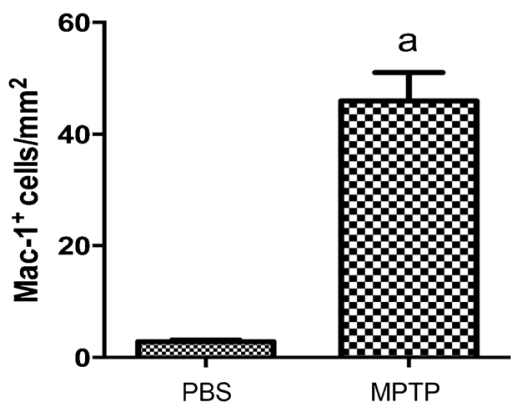

Fig. 4 Assessment of microglial reactivity following MPTP intoxication and LBT-3627 pretreatment. (A) Representative photomicrographs of Mac- $1^{+}$microglia within the substantia nigra of mice treated with PBS alone or at day 2 after MPTP intoxication ( $4 \times$ with $40 \times$ inset). Top right panel depicts number of reactive microglia taken from midbrains 2 days following MPTP treatment. Nigral sections were immunostained for Mac- $1^{+}$microglia using an anti-Mac-1 antibody, horse-conjugated secondary antibody, and diaminobenzidine. Numbers of reactive microglia were assessed by stereological analysis. (B) Photomicrographs of reactive microglia at day 7 after MPTP intoxication, followed by quantification of Mac- $1^{+}$cells $/ \mathrm{mm}^{2}$. Differences in means ( \pm SEM, $n=5-8$ ) were determined by analysis of variance followed by Fisher's least significant difference post hoc test where $p<0.05$ compared with groups treated with PBS (a) development of these agents and have entered into randomized clinical evaluations (NCT01882010). All such efforts have shown promise but with known limitations.

One such limitation rests in uncovering suitable biomarkers to track disease and therapeutic effectiveness. While immune monitoring of circulating blood cell numbers and functions have proven helpful, these tests are cumbersome, time consuming, and require multiple technical arms for evaluation and confirmatory assessments. Thus, the need for suitable biomarkers to track disease and therapeutic efficacy are real and timely. Here, we report that MEMRI can be used to assess inflammatory activities related to neuronal loss and astrocyte activation. With a neurotherapeutic directive in mind, our imaging results suggest the ability of MEMRI to detect immune modulation following MPTP treatment and LBT-3627 pretreatment. In this case, inflammation reduction seemed to outweigh the neuronal protection effect, possibly leading to lower MEMRI enhancement in LBT-3627 treated mice compared with MPTP mice.

$\mathrm{Mn}^{2+}$ serves as a surrogate measure of calcium influx linking MEMRI signal to the accumulation of the ion in neurons in an activity-dependent manner. As such, MEMRI signal is directly related to neural viability and activity [44,45]. To date, some studies have utilized MEMRI for studying pathobiology of neurodegenerative diseases, such as PD [46-49]. However, neurodegeneration is a dynamic process rather than a static event. Immune responses, neuronal impairment, and other pathological events are involved in 

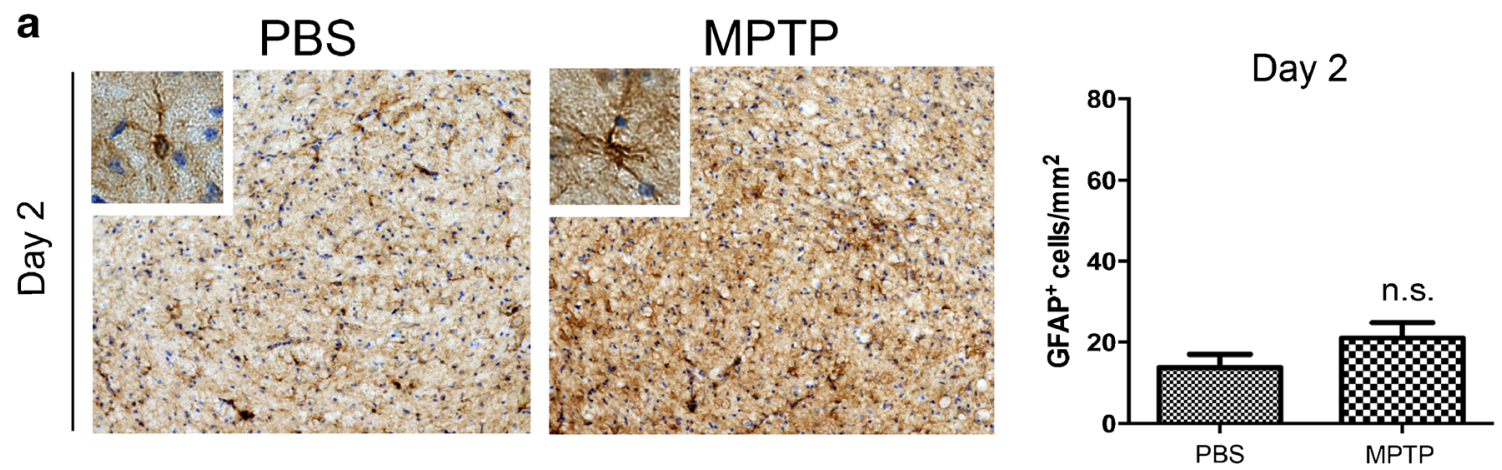

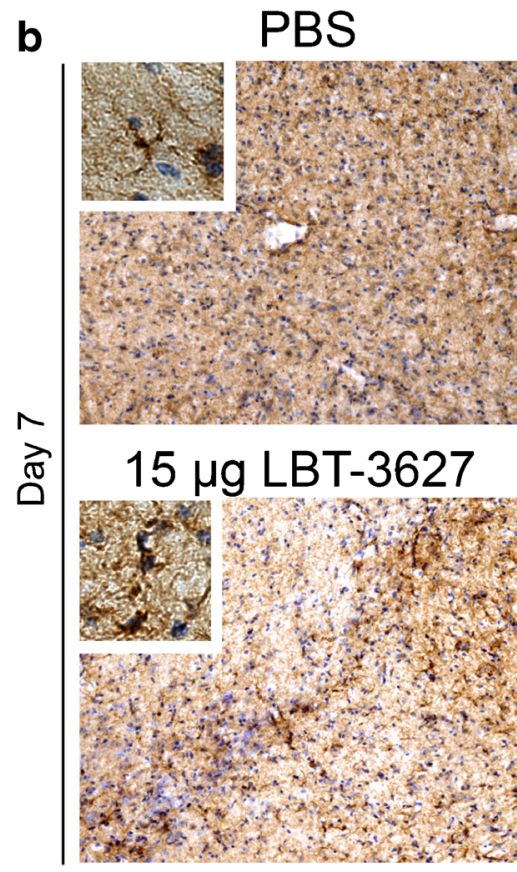

Fig. 5 Glial fibrillary acidic protein (GFAP) reactivity following MPTP intoxication and LBT-3627 pretreatment. (A) Representative photomicrographs of GFAP immunoreactive cells within the substantia nigra of mice (10× with $40 \times$ inset) treated with PBS or MPTP. Quantification of cell number is illustrated in the top right panel. (B) Photomicrographs of $\mathrm{GFAP}^{+}$cells following MPTP intoxication and LBT-3627 pretreatment at day 7. Quantification of day-7 reactive astrocytes follows. Serial
MPTP
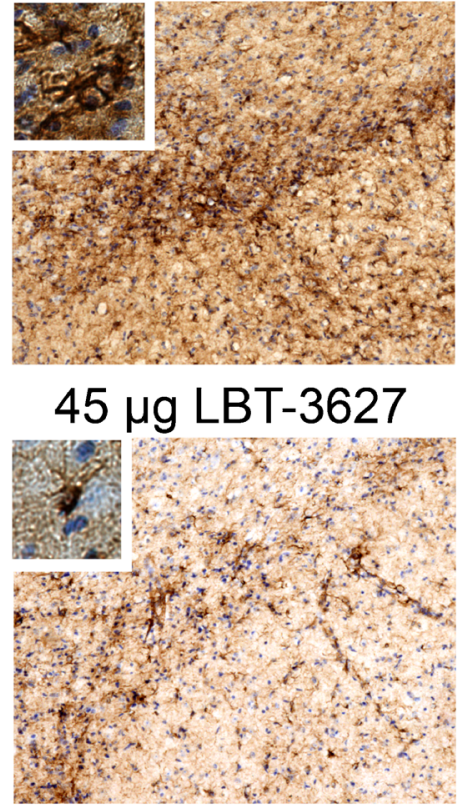

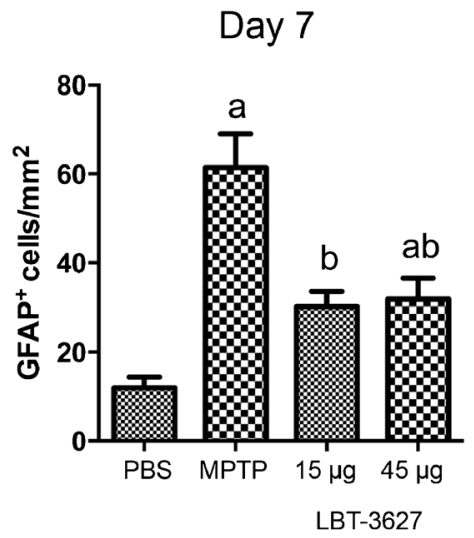

sections were stained with a primary antibody against GFAP, followed by a biotinylated secondary antibody, and diaminobenzidine color development. Sections were counterstained with thionin, and quantification was assessed using stereological analysis. Differences in means ( \pm SEM, $n=5-8$ ) were determined by analysis of variance followed by Fisher's least significant difference post hoc test where $p<0.05$ compared with groups treated with PBS (a) and MPTP (b)

neurodegenerative diseases, which can ultimately affect MEMRI signal intensity output. Delineation of the cellular basis of MEMRI is critical if it is harnessed as an imaging biomarker to gauge disease progression in experimental models. Previously, MEMRI signal change following a neuroinflammatory lesion was explored in a prior study from our laboratory [41]. MEMRI's ability to detect astrocyte, microglia and neuronal activation were measured following treatment with lipopolysaccharide or proinflammatory cytokines, both in vitro and in vivo. We were able to distinguish differences in MEMRI signal from either activated glia or neuronal injury. As such, we used the agent as a marker for neural activity in inflammatory and degenerative disease states. We also found axonal loss detected by traditional neurofilament staining coupled with MEMRI signal loss [35]. These findings support the notion that MEMRI reflects neuronal excitotoxicity and impairment that can occur through a range of insults including neuroinflammation. We concluded that the MEMRI signal enhancement is induced by inflammation stimulating neuronal $\mathrm{Mn}^{2+}$ uptake.

As previously stated, $\mathrm{Mn}^{2+}$ ions enter neurons through $\mathrm{Ca}^{2+}$ channels, and brain function depends on complex metabolic interactions amongst only a few different cell types, with astrocytes providing critical support for neuron function and survival. Astrocyte functions include buffering toxins in its extracellular environment, providing support to neurons, 


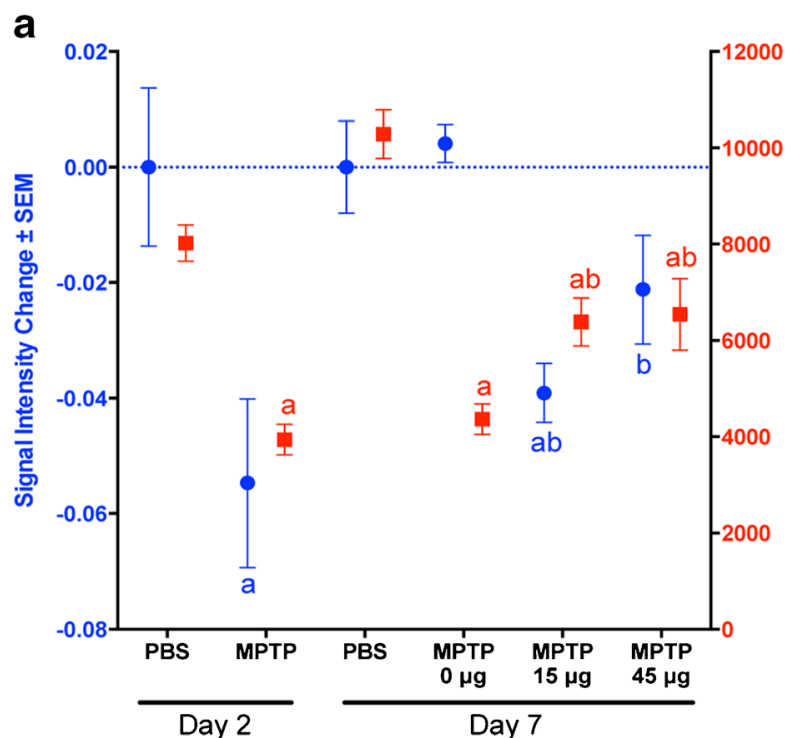

Day 2

b

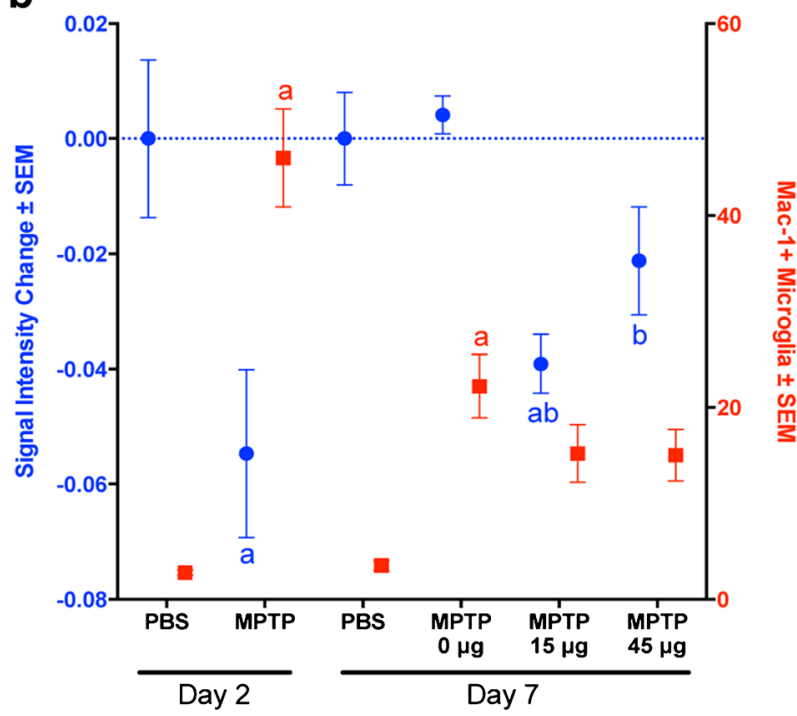

C

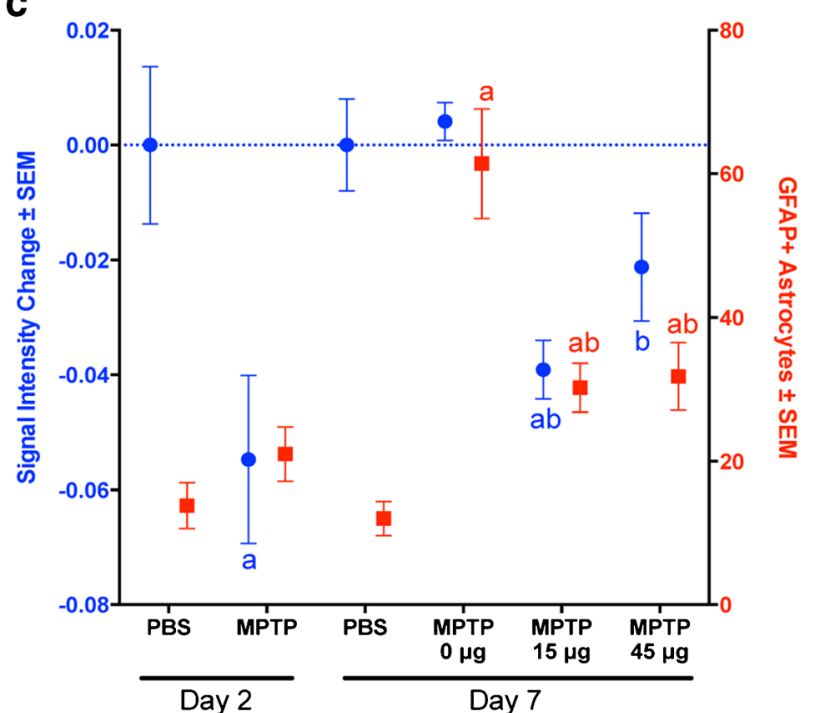

Fig. 6 Comparison of day-2 and day-7 immunohistology and signal enhancement. Change in signal intensity \pm SEM was determined from PBS controls and was compared with (A) total number of tyrosine hydroxylase $(\mathrm{TH})^{+}$neuron, (B) numbers of glial fibrillary acidic protein $\left(\right.$ GFAP) ${ }^{+}$astrocytes $/ \mathrm{mm}^{2}$, and (C) numbers of Mac- $1^{+}$microglia $/ \mathrm{mm}^{2}$ at days 2 and 7 following treatment with either MPTP or LBT-3627 $(0 \mu \mathrm{g}$, $15 \mu \mathrm{g}$, or $45 \mu \mathrm{g}$ ). Change in signal intensity is labeled in blue and cells numbers in red. Differences in means ( \pm SEM, $n=5-8$ ) were determined by analysis of variance followed by Fisher's least significant difference post hoc test where $p<0.05$ compared with groups treated with (a) PBS and (b) MPTP

modulating glutamate and glutamine, and affecting neuronal synaptic transmission [50,51]. Neurons undergo extremely oxidative metabolism making them highly susceptible to injury, whereas astrocytes are affected by glycolytic metabolism and are much less susceptible to oxygen deprivation. These differences in metabolism provide therapeutic opportunities to address $\mathrm{Mn}^{2+}$ sensitivity. Interactions between both cell types changes the metabolism of neurons through a range of mechanisms, including active glycolysis, lactate extrusion, and glutamate uptake by dysregulation of transporters [52,53]. Thus, activated astrocytes have a profound influence on neuronal metabolic susceptibilities and are changed by environment, disease, and therapeutic interventions that can alter function and lead to enhanced proliferation and cell surface receptor expression. In turn, the astrocyte phenotypic shift affects neuronal function. In support of this idea, in our prior study, we observed elevated $\mathrm{Mn}^{2+}$ uptake by neurons caused by astrogliosis [41]. Thus, we also posit that reactive astrocytes release cytokines stimulating neuronal $\mathrm{Ca}^{2+}$ channel activity and, ultimately, $\mathrm{Mn}^{2+}$ uptake. We demonstrated that MEMRI signals are increased during neuroinflammation owing to elevated neuronal $\mathrm{Mn}^{2+}$ uptake and accumulation through astrocyte activation through calcium channels [35,41]. Likewise, as $\mathrm{Mn}^{2+}$ is rapidly taken up into both neurons and astrocytes, neuronal death leads to the release of $\mathrm{Mn}^{2+}$, and leads to decreased MEMRI signal [40]. Similarly, in a model of traumatic brain injury, regions of MEMRI hyperintensity are seen at lesion sites and are associated with increased accumulation and infiltration of astrocytes to the lesion [54].

Similar effects can be observed in the current study at 2 days following MPTP intoxication, a time of peak neuronal loss and peak microglial response within the SN. Neuronal injury often leads to proliferation and migration of activated microglia to assist with cellular debris cleanup [55]. It could be hypothesized that activated microglia are ingesting released $\mathrm{Mn}^{2+}$ during this period, potentially leading to an increase in signal. However, we have previously found that macrophage uptake of $\mathrm{Mn}^{2+}$ using inductively coupled plasma mass spectrometry is not operative in these conditions (unpublished data). In accordance with this previous finding, increased early microgliosis appears to have a minimal effect on MEMRI enhancement at day 2, 
even with peak reactivity. Yet, there is the possibility that enhanced microglial reactivity could potentially be inhibiting neuronal uptake of $\mathrm{Mn}^{2+}$ due to elevated inflammatory states and neuronal degeneration, adding to a decrease in MEMRI signal. Interestingly, there was a significant signal enhancement found in the striatum of mice treated with MPTP rather than a decrease like that observed in the SN. This finding was not confirmed by immunohistology, but may be owing to the fact that striatal termini begin to degenerate prior to the loss of dopaminergic cell bodies [56]. Early degeneration of termini may induce astroglial reactivity more quickly than is seen in the SN, resulting in an increased signal enhancement. By 7 days after initial MPTP intoxication, $\mathrm{TH}^{+}$neuronal death is dominant, with limited microgliosis and a robust astroglial response. Treatment with LBT-3627 significantly attenuates the astroglial response, paralleled by a coincident decrease in signal enhancement. The results suggest that MEMRI signal enhancements can reflect both neuronal loss and reduced astrogliosis seen following therapeutic activities. Nonetheless, at day 7, MEMRI signal shows no differences between saline-treated controls and MPTPintoxicated mice. This seemingly discordant result can be explained by the astrocyte signal. As shown previously, astrocyte activation elicits MEMRI signal enhancement $[35,41]$. Moreover, astrogliosis and neurodegeneration can occur at the same time but have opposite effects on the MEMRI signal. That is, neuronal death elicits a signal decrease, while astrocytic activation enhances the signal. Interestingly, certain areas in hippocampus and cerebral cortex were found to be significantly increased in MEMRI enhancement in MPTP-treated mice on day 2, and lower enhancement in 15- $\mu$ g LBT-3627-treated mice compared with MPTP-treated mice on day 7 . In future studies, we will identify these areas and investigate the pathology underlying the MEMRI signal changes.

Relationships between MEMRI signal enhancement and glial interactions has been shown in a range of neurological disease models $[49,54,57,58]$. However, results have been conflicting. This has suggested that only astrocyte responses affect signal enhancement. Others have found that both activated microglia and astrocytes elicit signal change [58-60], or that astrocyte activation may play no role at all [57]. Thus, we chose to utilize an inflammatory model of disease that would allow us to evaluate longitudinally and independently microgliosis and astrocytic engagement in the MEMRI responses [8]. By assessing early events (2 days after MPTP intoxication), a time of peak inflammation and microglial reactivity coincident with a peak rate of neuronal death, we could assess signal decreases primarily linked to neuronal loss. Here, astrocyte involvement was not observed [8]. However, after 7 days, when microglial activation is diminished and nervous tissue repair is operative, coincident with astrocyte activation and facilitated $\mathrm{Mn}^{2+}$ uptake, MEMRI signal enhancement appears to be associated with changes in $\mathrm{GFAP}^{+}$reactivity. Such events are observed during treatment of MPTP intoxication. Indeed, we demonstrate that inflammationinduced astrocyte activation can stimulate $\mathrm{Mn}^{2+}$ uptake, potentially leading to MEMRI signal enhancement. However, the sensitivity of the MEMRI signal changes in relation to the changes in immunohistology is relatively low. The moderately low spatial resolution of MEMRI $\left(100 \mu \mathrm{m}^{3}\right.$ pixel size) compared with histological slices is one potential cause of the low sensitivity observed. Another reason can be attributed to the calculated $T_{1}$ wt signal enhancement as the measure of tissue $\mathrm{Mn}^{2+}$ concentration. For accurate calculation of MEMRI enhancement, scanner system variations among imaging sessions must be corrected, as described in a previous study [35]. Currently, we are developing 3-dimensional high resolution $\left(<100 \mu \mathrm{m}^{3}\right.$ pixel size) $\mathrm{T}_{1}$ mapping methods for future use, potentially providing improved assessment of underlying histopathology. Nonetheless, MEMRI is a valuable tool as it provides in vivo imaging examination with minimal invasiveness. Collectively, our results confirm the ability of MEMRI to detect neurodegeneration in areas of inflammation in an animal model, as well as monitor the success of anti-inflammatory and/ or immune modulating therapies in vivo. MEMRI can be used to identify brain subregions where inflammation is active and may also be useful for longitudinal measurement of inflammation in rodent models of neuroinflammatory disease, providing measurement tools to evaluate putative neurotherapeutics.

Acknowledgments This work was supported, in part, by the University of Nebraska Foundation, which includes donations from the Carol Swarts, M.D. Emerging Neuroscience Research Laboratory, the Margaret R. Larson Professorship, and the Frances and Louie Blumkin and Harriet Singer Endowment Funds, the Vice Chancellor's Office of the University of Nebraska Medical Center for Core Facility Developments, the Michael J. Fox Foundation, and National Institutes of Health grants P01 DA028555, R01 NS36126, P01 NS31492, 2R01 NS034239, P01 MH64570, P01 NS43985, P30 MH062261, R01 AG043540, and R01 NS070190 (R.L.M.). We would also like to acknowledge Barbara Clark for preparation of LBT-3627.

Required Author Forms Disclosure forms provided by the authors are available with the online version of this article.

\section{Compliance with Ethical Standards}

Conflict of Interest S. J. S. is an employee of Longevity Biotech, Inc., which is developing LBT-3627 as a clinical candidate. 


\section{References}

1. Ha D, Stone DK, Mosley RL, Gendelman HE. Immunization strategies for Parkinson's disease. Parkinsonism Relat Disord 2012;18(Suppl. 1):S218-S221.

2. Kosloski LM, Ha DM, Hutter JA, et al. Adaptive immune regulation of glial homeostasis as an immunization strategy for neurodegenerative diseases. J Neurochem 2010;114(5):1261-1276.

3. Mosley RL, Benner EJ, Kadiu I, et al. Neuroinflammation, oxidative stress and the pathogenesis of Parkinson's disease. Clin Neurosci Res 2006;6(5):261-281.

4. Benner EJ, Banerjee R, Reynolds AD, et al. Nitrated alphasynuclein immunity accelerates degeneration of nigral dopaminergic neurons. PLoS One 2008;3(1):e1376.

5. Fahn S. Description of Parkinson's disease as a clinical syndrome. Ann N Y Acad Sci 2003;991:1-14.

6. McGeer PL, Itagaki S, Akiyama H, McGeer EG. Rate of cell death in parkinsonism indicates active neuropathological process. Ann Neurol 1988;24(4):574-576.

7. McGeer PL, Itagaki S, Boyes BE, McGeer EG. Reactive microglia are positive for HLA-DR in the substantia nigra of Parkinson's and Alzheimer's disease brains. Neurology 1988;38(8):1285-1291.

8. Kohutnicka M, Lewandowska E, Kurkowska-Jastrzebska I, Czlonkowski A, Czlonkowska A. Microglial and astrocytic involvement in a murine model of Parkinson's disease induced by 1-methyl-4-phenyl-1,2,3,6-tetrahydropyridine (MPTP). Immunopharmacology 1998;39(3):167-180.

9. Kurkowska-Jastrzebska I, Wronska A, Kohutnicka M, Czlonkowski A, Czlonkowska A. The inflammatory reaction following 1-methyl-4-phenyl-1,2,3, 6-tetrahydropyridine intoxication in mouse. Exp Neurol 1999;156(1):50-61.

10. Yasuda Y, Shimoda T, Uno K, et al. The effects of MPTP on the activation of microglia/astrocytes and cytokine/chemokine levels in different mice strains. J Neuroimmunol 2008;204(1-2):43-51.

11. Reale $\mathrm{M}$, Iarlori $\mathrm{C}$, Thomas $\mathrm{A}$, et al. Peripheral cytokines profile in Parkinson's disease. Brain Behav Immun 2009;23(1):55-63.

12. Reynolds AD, Stone DK, Hutter JA, Benner EJ, Mosley RL, Gendelman HE. Regulatory T cells attenuate Th17 cell-mediated nigrostriatal dopaminergic neurodegeneration in a model of Parkinson's disease. J Immunol 2010;184(5):2261-2271.

13. Kosloski LM, Kosmacek EA, Olson KE, Mosley RL, Gendelman HE. GM-CSF induces neuroprotective and anti-inflammatory responses in 1-methyl-4-phenyl-1,2,3,6-tetrahydropyridine intoxicated mice. J Neuroimmunol 2013;265(1-2):1-10.

14. Huang X, Reynolds AD, Mosley RL, Gendelman HE. CD 4+ T cells in the pathobiology of neurodegenerative disorders. $\mathrm{J}$ Neuroimmunol 2009;211(1-2):3-15.

15. Bas J, Calopa M, Mestre M, et al. Lymphocyte populations in Parkinson's disease and in rat models of parkinsonism. J Neuroimmunol 2001;113(1):146-152.

16. Brochard V, Combadiere B, Prigent A, et al. Infiltration of CD4+ lymphocytes into the brain contributes to neurodegeneration in a mouse model of Parkinson disease. J Clin Invest 2009;119(1):182192.

17. Romero-Ramos M, von Euler Chelpin M, Sanchez-Guajardo V. Vaccination strategies for Parkinson disease: induction of a swift attack or raising tolerance? Hum Vaccin Immunother 2014;10(4): 852-867.

18. Saunders JA, Estes KA, Kosloski LM, et al. CD4+ regulatory and effector/memory $\mathrm{T}$ cell subsets profile motor dysfunction in Parkinson's disease. J Neuroimmune Pharmacol 2012;7(4):927938.
19. Olson KE, Kosloski-Bilek LM, Anderson KM, et al. Selective VIP receptor agonists facilitate immune transformation for dopaminergic neuroprotection in MPTP-intoxicated mice. J Neurosci 2015;35(50):16463-16478.

20. Schabitz WR, Kruger C, Pitzer C, et al. A neuroprotective function for the hematopoietic protein granulocyte-macrophage colony stimulating factor (GM-CSF). J Cereb Blood Flow Metab 2008;28(1): 29-43.

21. Delgado M, Pozo D, Ganea D. The significance of vasoactive intestinal peptide in immunomodulation. Pharmacol Rev 2004;56(2): 249-290.

22. Deng S, Xi Y, Wang H, Hao J, Niu X, Li W, et al. Regulatory effect of vasoactive intestinal peptide on the balance of Treg and Th17 in collagen-induced arthritis. Cell Immunol 2010;265(2):105-110.

23. Vial T, Descotes J. Clinical toxicity of cytokines used as haemopoietic growth factors. Drug Saf 1995;13(6):371-406.

24. Kwakye GF, Paoliello MM, Mukhopadhyay S, Bowman AB, Aschner M. Manganese-induced parkinsonism and Parkinson's disease: shared and distinguishable features. Int J Environ Res Public Health 2015;12(7):7519-7540.

25. Olanow CW. Manganese-induced parkinsonism and Parkinson's disease. Ann N Y Acad Sci 2004;1012:209-223.

26. Jackson-Lewis V, Przedborski S. Protocol for the MPTP mouse model of Parkinson's disease. Nat Protoc 2007;2(1):141-151.

27. Przedborski S, Jackson-Lewis V, Naini AB, et al. The parkinsonian toxin 1-methyl-4-phenyl-1,2,3,6-tetrahydropyridine (MPTP): a technical review of its utility and safety. J Neurochem 2001;76(5): 1265-1274.

28. Grunecker B, Kaltwasser SF, Peterse Y, et al. Fractionated manganese injections: effects on MRI contrast enhancement and physiological measures in C57BL/6 mice. NMR Biomed 2010;23(8):913921.

29. Grunecker B, Kaltwasser SF, Zappe AC, et al. Regional specificity of manganese accumulation and clearance in the mouse brain: implications for manganese-enhanced MRI. NMR Biomed 2013;26: 542-556.

30. Koretsky AP, Silva AC. Manganese-enhanced magnetic resonance imaging (MEMRI). NMR Biomed 2004;17(8):527-531.

31. Silva AC, Bock NA. Manganese-enhanced MRI: an exceptional tool in translational neuroimaging. Schizophr Bull 2008;34(4): 595-604.

32. Silva AC, Lee JH, Aoki I, Koretsky AP. Manganese-enhanced magnetic resonance imaging (MEMRI): methodological and practical considerations. NMR Biomed 2004;17(8):532-543.

33. Lee JH, Silva AC, Merkle H, Koretsky AP. Manganese-enhanced magnetic resonance imaging of mouse brain after systemic administration of $\mathrm{MnCl} 2$ : dose-dependent and temporal evolution of $\mathrm{T} 1$ contrast. Magn Reson Med 2005;53(3):640-648.

34. Bock NA, Paiva FF, Silva AC. Fractionated manganese-enhanced MRI. NMR Biomed 2008;21(5):473-478.

35. Bade AN, Gorantla S, Dash PK, et al. Manganese-enhanced magnetic resonance imaging reflects brain pathology during progressive HIV-1 infection of humanized mice. Mol Neurobiol 2016 Jul;53(5): 3286-3297.

36. Benner EJ, Mosley RL, Destache CJ, et al. Therapeutic immunization protects dopaminergic neurons in a mouse model of Parkinson's disease. Proc Natl Acad Sci U S A 2004;101(25): 9435-9440.

37. Jackson-Lewis V, Jakowec M, Burke RE, Przedborski S. Time course and morphology of dopaminergic neuronal death caused by the neurotoxin 1-methyl-4-phenyl-1,2,3,6-tetrahydropyridine. Neurodegeneration 1995;4(3):257-269. 
38. Inoue T, Majid T, Pautler RG. Manganese enhanced MRI (MEMRI): neurophysiological applications. Rev Neurosci 2011;22(6):675-694.

39. Eschenko O, Canals S, Simanova I, Beyerlein M, Murayama Y, Logothetis NK. Mapping of functional brain activity in freely behaving rats during voluntary running using manganese-enhanced MRI: implication for longitudinal studies. NeuroImage 2010;49(3):2544-2555.

40. Massaad CA, Pautler RG. Manganese-enhanced magnetic resonance imaging (MEMRI). Methods Mol Biol 2011;711:145-174.

41. Bade AN, Zhou B, Epstein AA, et al. Improved visualization of neuronal injury following glial activation by manganese enhanced MRI. J Neuroimmune Pharmacol 2013;8(4):1027-1036.

42. Olanow CW, Stern MB, Sethi K. The scientific and clinical basis for the treatment of Parkinson disease (2009). Neurology 2009;72(21 Suppl. 4):S1-S136.

43. Olson KE, Gendelman HE. Immunomodulation as a neuroprotective and therapeutic strategy for Parkinson's disease. Curr Opin Pharmacol 2016;26:87-95.

44. Pautler RG, Koretsky AP. Tracing odor-induced activation in the olfactory bulbs of mice using manganese-enhanced magnetic resonance imaging. NeuroImage 2002;16(2):441-448.

45. Takeda A, Devenyi A, Connor JR. Evidence for non-transferrinmediated uptake and release of iron and manganese in glial cell cultures from hypotransferrinemic mice. J Neurosci Res 1998;51(4):454-462.

46. Weng JC, Tikhonova MA, Chen JH, et al. Ceftriaxone prevents the neurodegeneration and decreased neurogenesis seen in a Parkinson's disease rat model: an immunohistochemical and MRI study. Behav Brain Res 2016;305:126-139.

47. Pelled G, Bergman H, Ben-Hur T, Goelman G. Manganeseenhanced MRI in a rat model of Parkinson's disease. J Magn Reson Imaging 2007;26(4):863-870.

48. Kondoh T, Bannai M, Nishino H, Torii K. 6-Hydroxydopamineinduced lesions in a rat model of hemi-Parkinson's disease monitored by magnetic resonance imaging. Exp Neurol 2005;192(1): 194-202.

49. Soria G, Aguilar E, Tudela R, Mullol J, Planas AM, Marin C. In vivo magnetic resonance imaging characterization of bilateral structural changes in experimental Parkinson's disease: a T2 relaxometry study combined with longitudinal diffusion tensor imaging and manganese-enhanced magnetic resonance imaging in the 6-hydroxydopamine rat model. Eur J Neurosci 2011;33(8):15511560.

50. Kimelberg HK, Nedergaard M. Functions of astrocytes and their potential as therapeutic targets. Neurotherapeutics 2010;7(4):338353.

51. Khakh BS, Sofroniew MV. Diversity of astrocyte functions and phenotypes in neural circuits. Nat Neurosci 2015;18(7):942-952.

52. Ricci G, Volpi L, Pasquali L, Petrozzi L, Siciliano G. Astrocyteneuron interactions in neurological disorders. J Biol Phys 2009;35(4):317-336.

53. Araque A. Astrocyte-neuron signaling in the brain - implications for disease. Curr Opin Invest Drugs 2006;7(7):619-624.

54. Talley Watts L, Shen Q, Deng S, Chemello J, Duong TQ. Manganese-enhanced magnetic resonance imaging of traumatic brain injury. J Neurotrauma 2015;32(13):1001-1010.

55. Neumann H, Kotter MR, Franklin RJ. Debris clearance by microglia: an essential link between degeneration and regeneration. Brain 2009; 132(Pt 2):288-295.

56. Burke RE, O'Malley K. Axon degeneration in Parkinson's disease. Exp Neurol 2013;246:72-83.

57. Immonen RJ, Kharatishvili I, Sierra A, Einula C, Pitkanen A, Grohn OH. Manganese enhanced MRI detects mossy fiber sprouting rather than neurodegeneration, gliosis or seizure-activity in the epileptic rat hippocampus. NeuroImage 2008;40(4):17181730.

58. Haapanen A, Ramadan UA, Autti T, Joensuu R, Tyynela J. In vivo MRI reveals the dynamics of pathological changes in the brains of cathepsin D-deficient mice and correlates changes in manganeseenhanced MRI with microglial activation. Magn Reson Imaging 2007;25(7):1024-1031.

59. Kawai Y, Aoki I, Umeda M, et al. In vivo visualization of reactive gliosis using manganese-enhanced magnetic resonance imaging. NeuroImage 2010;49(4):3122-3131.

60. Wideroe M, Olsen O, Pedersen TB, et al. Manganese-enhanced magnetic resonance imaging of hypoxic-ischemic brain injury in the neonatal rat. NeuroImage 2009;45(3):880-890. 